\title{
Degenerate Parabolic Differential Equations
}

By

\author{
Katsuju IGARI*
}

\section{§1. Introduction}

We are concerned with the Cauchy problem for the equation

$$
\begin{aligned}
& \partial_{t} u-\sum_{j, k=1}^{n} \partial_{x_{j}}\left(a_{j k}(x, t) \partial_{x_{k}} u\right)-\sum_{j=1}^{n} b_{j}(x, t) \partial_{x_{\jmath}} u-c(x, t) u \\
& =\partial_{t} u-A u=f
\end{aligned}
$$

$(x, t)$ in $\mathbb{R}^{n} \times[0, \infty)$ with the initial datum

$$
u(x, 0)=u_{0}(x)
$$

Throughout this paper we use the following abbreviation: $\partial_{t}, \partial_{x_{j}}$ stands for $\frac{\partial}{\partial t}, \frac{\partial}{\partial x_{j}}$ respectively. We assume that $a_{j k}(x, t), b_{j}(x, t), c(x, t)$ are real-valued smooth functions. Moreover $\left(a_{j k}\right)_{1 \leqq j \leqq n, 1 \leqq k \leqq n}$ is supposed to be symmetric and to satisfy the following condition: for any $(x, t)$ in $\mathbb{R}^{n} \times[0, \infty)$

$$
\operatorname{Re} \sum_{j, k=1}^{n} a_{j k}(x, t) \xi_{j} \xi_{k} \geqq 0 \quad \text { for all } \xi \in \mathbb{R}^{n} .
$$

O.A. Oleinik has treated this problem (see [4], [5]). Her method consists of the following procedure (elliptic regularization): instead of (1.1), the following equations (depending on a positive parameter $\varepsilon$ ) in $G=\mathbb{R}^{n} \times[0, T]$

$$
\partial_{i} u-\varepsilon \Delta u-A u=f
$$

are considered. Let $u_{\varepsilon}$ be the solution of (1.4) with the given initial value $u_{0}(x) \in L^{2}\left(\mathbb{R}^{n}\right)$ and $f(x, t) \in L^{2}(G)$. Then it is shown that $\left\{u_{\varepsilon}(x, t)\right\}$ is

Communicated by S. Matsuura, March 17, 1973.

* Department of Mathematics, Kyoto University, Kyoto. 
bounded in $L^{2}(G)$. Then a weak limit of them, as $\varepsilon \rightarrow 0$, gives the desired weak solution $u(x, t) \in L^{2}(G)$. The uniqueness of the solution is proved. She also proved the smoothness of $u$, assuming the smoothness of $u_{0}$ and $f$.

Contrary to the above point of view, we regard (1.1) as evolution equation. More precisely, we want to show the existence of the unique solution $u(x, t) \in \mathscr{E}_{t}^{0}\left(L^{2}\right) \cap \mathscr{E}_{t}^{1}\left(\mathscr{D}_{L^{2}}^{\prime 2}\right)$ of $(1.1)-(1.2)$ for any initial value $u_{0}(x) \in L^{2}$ and any $f(x, t) \in \mathscr{E}_{t}^{0}\left(L^{2}\right) * *$

Our approach is based on the semi-group theory. Instead of elliptic regularization, we use Friedrichs' mollifier. Its property (Lemma 1) gives immediately the desired result (Theorem 1). It also gives the energy inequality (Proposition 1), which seems to be important. But the inequality of this form is not stated in [4], [5]. The smoothness of the solution can be obtained in the following form (Theorem 2): when $u_{0}(x) \in \mathscr{D}_{L^{2}}^{m}$ and $f(x, t) \in \mathscr{E}_{t}^{0}\left(\mathscr{D}_{L^{2}}^{m}\right)$, the solution $u(x, t)$ belongs to $\mathscr{E}_{t}^{0}\left(\mathscr{D}_{L^{2}}^{m}\right) \cap \mathscr{E}_{t}^{1}\left(\mathscr{D}_{L^{2}}^{m-2}\right)$. We study also the case where $b_{j}(x, t)$ and $c(x, t)$ are complex-valued functions (Remark in the section 3 ).

It seems to us that our method is more natural than the one relying on elliptic regularization and will be useful to other problems. $\left.{ }^{* *}\right)$

\section{§2。 Emergy Inequality}

Let $a_{j k}(x, t) \in \mathscr{E}_{t}^{0}\left(\mathscr{B}^{2}\right) ; b_{j}(x, t) \in \mathscr{E}_{t}^{0}\left(\mathscr{B}^{1}\right) ; c(x, t) \in \mathscr{E}_{t}^{0}\left(\mathscr{B}^{0}\right)$. We assume the condition (1.3). Then we have the following proposition.

$\mathbb{P}_{\text {roposition }} \mathbb{1}$ (Ermergy ingequality)。 Let $f(x, t) \in \mathscr{E}_{t}^{0}\left(L^{2}\right)$ and $u(x, t) \in \mathscr{E}_{t}^{0}\left(L^{2}\right) \cap \mathscr{E}_{t}^{1}\left(\mathscr{D}_{L^{2}}^{2}\right)$ be a solution of (1.1). Then it holds for any

*) In this paper, we use the following notation: $x=\left(x_{1}, \ldots, x_{n}\right) . \quad \partial_{r}^{\nu}=\partial_{r_{1}}^{\nu} \ldots \partial_{x n}^{\nu}, \quad \nu=$ $\left(\nu_{1}, \ldots, \nu_{n}\right) . L^{2}=L^{2}\left(\mathbb{R}^{n}\right) . \quad u(x) \in \mathscr{D}_{L^{2}}^{m}$ means that its derivatives $\partial_{x}^{\nu} u$ (in the sense of distribution) up to order $m$ belong to $L^{2}$. $\mathscr{D}_{L^{2}}^{m}$ provided with the scalar product $(u, v)_{m}=\sum_{|\nu| \leqq m}\left(\partial_{x}^{\nu} u, \partial_{x}^{\nu} v\right)$ is a Hilbert space. $\mathscr{D}_{L^{2}}^{\prime m}$ is the dual space of $\mathscr{D}_{L^{2}}^{m}$ and sometimes we denote it by $\mathscr{D}_{L^{2}}^{m} . \quad \varphi(x) \in \mathscr{B}^{m}$ means that its derivatives $\partial_{x}^{\nu} \varphi$ up to order $m$ are continuous and bounded in $\mathbb{R}^{n} . \varphi(x) \in \mathscr{D}$ means that $\varphi(x)$ is infinitely differentiable and has compact support. $f(t) \in \mathscr{E}_{t}^{k}\left(\mathscr{D}_{L^{2}}^{m}{ }_{\mathrm{o}}\left(\right.\right.$ or $\left.\left.\mathscr{B}^{m}\right)\right)$ means that $t \rightarrow f(t) \in \mathscr{D}_{L^{2}}^{m}$ (or $\mathscr{B}^{m}$ ) is continuously differentiable up to order $k$.

**) The summary of the results obtained below was announced in the following paper: Cauchy problem for degenerate parabolic equations, Proc. J. Acad., 49 (1973), 229-232. 
$t(0 \leqq t \leqq T)$ that

$$
\|u(t)\| \leqq e^{\gamma t}\|u(0)\|+\int_{0}^{t} e^{\gamma(t-s)}\left\|_{1} f(s)\right\| d s
$$

where $\gamma$ is a constant which may depend on $T$ but does not depend on $u$ and $f$.

To see this, the following lemma is essential.

Lermma 耳. Let $\rho_{\varepsilon} *$ be Friedrichs' mollifier, where we assume $\rho(x)$ even function. Let $a(x) \in \mathscr{B}^{2}$ be real-valued function, and let $u(x) \in L^{2}$. Then it holds for any $\nu(|\nu| \leqq 2)$ that

1) $\left|\operatorname{Re}\left(u_{\varepsilon},\left[\rho_{\varepsilon}^{*}, a(x)\right] \partial_{x}^{\nu} u\right)\right| \leqq C\|u\|$,

2) $\operatorname{Re}\left(u_{\varepsilon},\left[\rho_{\varepsilon} *, a(x)\right] \partial_{x}^{\nu} u\right) \rightarrow 0 \quad$ as $\varepsilon \rightarrow 0$,

where $u_{\varepsilon}$ stands for $\rho_{\varepsilon} * u,\left[\rho_{\varepsilon}^{*}, a(x)\right] \partial_{x}^{\nu} u=\rho_{\varepsilon} *\left\{a(x) \partial_{x}^{\nu} u\right\}-u(x) \rho_{\varepsilon} *\left(\partial_{x}^{\nu} u\right)$, and $C$ is a constant independent of $u$ and $\varepsilon$.

Proof of Lemma 1. Consider only the case of $|\nu|=2$, because, in the case of $|\nu| \leqq 1,1)$ and 2) are clear by Friedrichs' lemma. We denote $\partial_{x}^{\nu}$ by $\partial_{x_{j}} \partial_{x_{k}}$. By Taylor expansion

$$
\begin{aligned}
& {\left[\rho_{\varepsilon} *, a(x)\right] \partial_{x_{\jmath}} \partial_{x_{k}} u=-\sum_{i=1}^{n} a^{(i)}(x)\left(x_{i} \rho_{\varepsilon}\right) * \partial_{x_{\jmath}} \partial_{x_{k}} u} \\
& \quad+\sum_{|\mu|=2} \frac{1}{\mu !} \int a_{\mu}(x, y)(x-y)^{\mu} \rho_{\varepsilon}(x-y) \partial_{y_{\jmath}} \partial_{y_{k}} u(y) d y .
\end{aligned}
$$

At first we take the 1-st term of (2.2).

$$
\begin{aligned}
& -2 \operatorname{Re}\left(u_{\varepsilon}, a^{(i)}(x)\left(x_{i} \rho_{\varepsilon}\right) * \partial_{x_{j}} \partial_{x_{k}} u\right)=\left(u^{(i, j)}(x)\left(x_{i} \rho_{\varepsilon}\right) * \partial_{x_{k}} u, u_{\varepsilon}\right) \\
& +\left(u_{\varepsilon},\left[\left(v_{\imath} \rho_{\varepsilon}\right) *, a^{(i)}(x)\right] \partial_{x_{j}} \partial_{x_{h}} u\right)+\left(u_{\varepsilon},\left(x_{\imath} \rho_{\varepsilon}\right) *\left\{a^{(i, k)}(x) \partial_{x_{j}} u\right\}\right) \\
& +\left(\left(x_{i} \rho_{\varepsilon}\right) * \partial_{x_{k}} u,\left[a^{(i)}(x), \rho_{\varepsilon} *\right] \partial_{x_{\jmath}} u\right),
\end{aligned}
$$

where we used the relations that $\left(\left(x_{i} \rho_{\varepsilon}\right) * u, v\right)=-\left(u,\left(x_{i} \rho_{\varepsilon}\right) * v\right)$ and that $\left(\rho_{\varepsilon} * u, v\right)=\left(u, \rho_{\varepsilon} * v\right)$. All these terms in the right-hand side of (2.3) can be majorized by

$$
\sum_{i, j=1}^{n}\|u\|\left\|\left(x_{i} \rho_{\varepsilon}\right) * \partial_{x_{j}} u\right\|+\sum_{\nu \mid \leqq 2} \sum_{i=1}^{n}\|u\|\left\|\left[a^{(i)}(x),\left(x_{\imath} \rho_{\varepsilon}\right) *\right] \partial_{x}^{\nu} u\right\| .
$$


In the same way as Friedrichs' lemma, we can show for any $\nu(|\nu| \leqq 2)$

$$
\begin{aligned}
& \left\|\left(x_{i} \rho_{\varepsilon}\right) * \partial_{x_{j}} u\right\|,\left\|\left[a^{(i)}(x),\left(x_{i} \rho_{\varepsilon}\right) *\right] \partial_{x}^{\nu} u\right\| \leqq \text { const. }\|u\|, \\
& \left\|\left(x_{i} \rho_{\varepsilon}\right) * \partial_{x_{j}} u\right\|,\left\|\left[a^{(i)}(x),\left(x_{i} \rho_{\varepsilon}\right) *\right] \partial_{x}^{\nu} u\right\| \rightarrow 0 \quad \text { as } \varepsilon \rightarrow 0 .
\end{aligned}
$$

Next we consider the 2-nd term of (2.2). Denote it by $R_{\varepsilon} u$.

$$
\begin{aligned}
R_{\varepsilon} u & =\sum_{|\mu|=2} \frac{1}{\mu !} \int \partial_{y_{j}} \partial_{y_{k}}\left\{a_{\mu}(x, y)(x-y)^{\mu} \rho_{\varepsilon}(x-y\} u(y) d y\right. \\
& =\sum_{|\mu|=2} \frac{1}{\mu !} \int \partial_{y_{j}} \partial_{y_{k}}\left\{a_{\mu}(x, y)(x-y)^{\mu} \rho_{\varepsilon}(x-y)\right\}\{u(y)-u(x)\} d y .
\end{aligned}
$$

If we note that $\sum_{|\nu| \leqq 2} \sum_{|\mu|=2} \int\left|\left(x^{\mu} \rho_{\varepsilon}\right)^{(\nu)}(x)\right| d x<$ const. (independent of $\varepsilon$ ), the same reasoning as in the proof of Friedrichs' lemma gives

$$
\begin{aligned}
& \left\|R_{\varepsilon} u\right\| \leqq \text { const. }\|u\|, \\
& R_{\varepsilon} u \rightarrow 0 \quad \text { as } \varepsilon \rightarrow 0 .
\end{aligned}
$$

If we use this lemma, we can prove Proposition 1 in the same way as hyperbolic equations (cf. [3], §2 in Chapter 6).

Proof of Proposition 1. Apply $\rho_{\varepsilon} *$ to (1.1) and consider $\frac{d}{d t}\left\|u_{\varepsilon}(t)\right\|^{2}$.

$$
\begin{aligned}
\frac{d}{d t}\left\|u_{\varepsilon}(t)\right\|^{2} & =2 \operatorname{Re}\left(u_{\varepsilon}(t), \frac{d}{d t} u_{\varepsilon}(t)\right) \\
& =2 \operatorname{Re}\left(u_{\varepsilon}(t), A u_{\varepsilon}(t)+\left[\rho_{\varepsilon} *, A\right] u(t)+f_{\varepsilon}(t)\right) .
\end{aligned}
$$

By the condition (1.3)

$$
\begin{aligned}
& 2 \operatorname{Re}\left(u_{\varepsilon}(t), \partial_{x_{j}}\left(a_{j_{k}} \partial_{x_{k}} u_{\varepsilon}(t)\right)\right)=-2\left(a_{j_{k}} \partial_{x_{k}} u_{\varepsilon}(t), \partial_{x_{j}} u_{\varepsilon}(t)\right) \leqq 0 . \\
& 2 \operatorname{Re}\left(u_{\varepsilon}(t), b_{j} \partial_{x_{j}} v_{\varepsilon}(t)\right)=-\left(u_{\varepsilon}(t), b_{j}^{(j)} u_{\varepsilon}(t)\right) \leqq \text { const. }\left\|u_{\varepsilon}(t)\right\|^{2} .
\end{aligned}
$$

Therefore we have

$$
\begin{aligned}
\frac{d}{d t}\left\|u_{\varepsilon}(t)\right\|^{2} \leqq 2 \gamma\left\|u_{\varepsilon}(t)\right\|^{2}+2\left\|u_{\varepsilon}(t)\right\|\left\|f_{\varepsilon}(t)\right\| \\
+2 \operatorname{Re}\left(u_{\varepsilon}(t),\left[\rho_{\varepsilon} *, A\right] u(t)\right) .
\end{aligned}
$$




\section{$\S 3 . \quad$ Existence Theorem}

At first we consider the case where the coefficients are functions of only $x$. Let $a_{j k}(x) \in \mathscr{B}^{2} ; b_{j}(x) \in \mathscr{B}^{1} ; c(x) \in \mathscr{B}^{0}$. We assume the condition (1.3). Then we have the following proposition which shows Hille-Yosida's theorem is applicable.

Proposition 2. Take the domain of definition $\mathscr{D}(A)$ of $A$ as follows:

$$
\mathscr{D}(A)=\left\{u(x) ; u(x) \in L^{2}, A u(x) \in L^{2}\right\} .
$$

Then, for large $\lambda,(\lambda-A)$ defines a one-to-one surjective mapping of $\mathscr{D}(A)$ onto $L^{2}$. Moreover there exists a constant $\beta$ such that

$$
\left\|(\lambda-A)^{-1}\right\|_{\mathscr{L}\left(L^{2}, L^{2}\right)} \leqq \frac{1}{\lambda-\beta} \quad \text { for any } \lambda>\beta
$$

Proof. For any $u \in \mathscr{D}(A)$ it holds that

$$
\|(\lambda-A) u\|^{2} \geqq\left(\lambda^{2}-\text { const. } \lambda\right)\|u\|^{2}+\|A u\|^{2} \text {. }
$$

Indeed

$$
\begin{aligned}
& \left\|\rho_{\varepsilon} *(\lambda-A) u\right\|^{2}=\lambda^{2}\left\|u_{\varepsilon}\right\|^{2}+\left\|\rho_{\varepsilon} * A u\right\|^{2}-2 \operatorname{Re}\left(u_{\varepsilon}, A u_{\varepsilon}+\left[\rho_{\varepsilon} *, A\right] u\right) \\
& \geqq\left(\lambda^{2}-\text { const. } \lambda\right)\left\|u_{\varepsilon}\right\|^{2}+\left\|\rho_{\varepsilon} * A u\right\|^{2}-2 \operatorname{Re}\left(u_{\varepsilon},\left[\rho_{\varepsilon} *, A\right] u\right) .
\end{aligned}
$$

By the passage to the limit, using Lemma 1, we have (3.3).

The inequality (3.3) shows that, for large $\lambda,(\lambda-A)$ defines a one-to-one closed mapping of $\mathscr{D}(A)$ into $L^{2}$. Therefore we have only to show that the image $(\lambda-A) \mathscr{D}(A)$ is dense in $L^{2}$. We show this by contradiction. If not dense, there exists $v(\neq 0)$ in $L^{2}$ such that

$$
((\lambda-A) u, v)=0 \quad \text { for every } u \in \mathscr{D}(A)
$$

Of course this holds for every $u \in \mathscr{D}$. Hence

$$
\left(\lambda-A^{*}\right) v=0 \quad \text { in the sense of distribution, }
$$


where $A^{*}$ is the formal adjoint of $A$.

Since $v \in L^{2}$, (3.4) shows that $A^{*} v$ belongs to $L^{2}$. If we note that $A^{*}$ satisfies the same condition as $A$, we can use the inequality (3.3) and have

$$
0=\left\|\left(\lambda-A^{*}\right) v\right\|^{2} \geqq\left(\lambda^{2}-\text { const. } \lambda\right)\|v\|^{2} \text {. }
$$

For large $\lambda$, this inequality requires that $v=0$. This is contradictory to our assumption $v \neq 0$.

Q.E.D.

The above proposition shows that all the conditions of Hille-Yosida's theorem are satisfied. Therefore we have

Corollary。 For any initial value $u_{0}(x) \in \mathscr{D}(A)$ and any $f(t)$ such that $f(t)$ and $A f(t)$ belong to $\mathscr{E}_{t}^{0}\left(\mathbb{L}^{2}\right)$, there exists a unique solution $u(x, t) \in$ $\mathscr{E}_{t}^{1}\left(L^{2}\right)$ of the Cauchy problem (1.1)-(1.2).

Now we consider the general case, i.e., the case where the coefficients are functions of $x$ and also of $t$. Let $a_{j k}(x, t) \in \mathscr{E}_{t}^{0}\left(\mathscr{B}^{2}\right) ; b_{j}(x, t) \in \mathscr{E}_{t}^{0}\left(\mathscr{B}^{1}\right)$; $c(x, t) \in \mathscr{E}_{t}^{0}\left(\mathscr{B}^{0}\right)$. We assume the condition (1.3). Then, using Proposition 1 and the above Corollary, we have the following theorem.

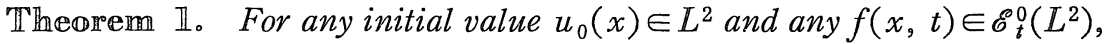
there exists a unique solution $u(x, t) \in \mathscr{E}_{t}^{0}\left(L^{2}\right) \cap \mathscr{E}_{t}^{1}\left(\mathscr{D}_{L^{2}}^{\prime 2}\right)$ of the Cauchy problem (1.1)-(1.2).

Proof. At first we show this in the case where the coefficients are functions of only $x$. Denote $\rho_{\varepsilon} * u_{0}$ and $\rho_{\varepsilon} * f(t)$ by $u_{0}^{\varepsilon}(x)$ and $f_{\varepsilon}(x, t)$ respectively. $u_{0}^{\varepsilon}(x)$ belongs to $\mathscr{D}(A)$ and $f_{\varepsilon}(t), A f_{\varepsilon}(t)$ belong to $\mathscr{E}_{t}^{0}\left(L^{2}\right)$. Hence, by Corollary, we see the existence of the unique solution $u_{\varepsilon}(x, t)$ $\in \mathscr{E}_{t}^{1}\left(L^{2}\right)$ of the Cauchy problem (1.1)-(1.2) with the initial value $u_{0}^{\varepsilon}(x)$ and with the right-hand term $f_{\varepsilon}(t)$.

Here we use the energy inequality (2.1) for $u_{\varepsilon}(t)-u_{\varepsilon^{\prime}}(t)$. Then

$$
\max _{0 \leqq t \leqq T}\left\|u_{\varepsilon}(t)-u_{\varepsilon^{\prime}}(t)\right\| \leqq M\left\{\left\|u_{0}^{\varepsilon}-u_{0}^{\varepsilon^{\prime}}\right\|+\max _{0 \leqq t \leqq T}\left\|f_{\varepsilon}(t)-f_{\varepsilon^{\prime}}(t)\right\|\right\},
$$

where $\mathbb{M}$ is a constant independent of $\varepsilon, \varepsilon^{\prime}$. Therefore $\left\{u_{\varepsilon}(x, t)\right\}$ is a Cauchy sequence in $\mathscr{E}_{t}^{0}\left(L^{2}\right), 0 \leqq t \leqq T$. We denote its limit by $u(x, t)$, $u(x, t) \in \mathscr{E}_{t}^{0}\left(L^{2}\right)$. On the other hand 


$$
u_{\varepsilon}(t)-u_{0}^{\varepsilon}=\int_{0}^{t}\left\{A u_{\varepsilon}(s)+f_{\varepsilon}(s)\right\} d s
$$

By the passage to the limit, as $\varepsilon \rightarrow 0$, we have

$$
u(t)-u_{0}=\int_{0}^{t}\{A u(s)+f(s)\} d s
$$

where the integral is taken in the sense of $\mathscr{D}_{L^{2}}^{2}$. Therefore we see that $t \rightarrow u(t) \in \mathscr{D}_{L^{2}}^{\prime 2}$ is continuously differentiable and that the equation

$$
\partial_{t} u-A u=f
$$

is satisfied in the sense of $\mathscr{D}_{L^{2}}^{\prime 2}$.

Next, we prove the theorem in the case where the coefficients are functions of $x$ and also of $t$. Denote $\mathbb{R}^{n} \times[0, T]$ by $G$. Let $0=t_{0} \leqq t_{1} \leqq \cdots$ $\leqq t_{k}=T$ be a subdivision of $[0, T]$ of equal length. We define $u_{1}(t)$, $u_{2}(t), \ldots, u_{k}(t)$ in $\left[t_{0}, t_{1}\right],\left[t_{1}, t_{2}\right], \ldots,\left[t_{k-1}, t_{k}\right]$ by the following condition:

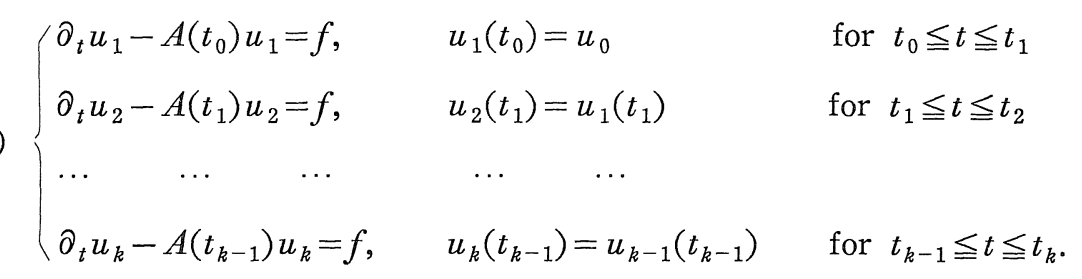

We denote by $u^{(k)}(t)$ the function which in $t_{j-1} \leqq t \leqq t_{j}$ is equal to $u_{j}(t)$.

It is easy to see that $\left\{u^{(k)}(t)\right\}$ is uniformly bounded in the space $\mathscr{E}_{[-2]}^{2,2}(G)$, consisting of all functions $u(x, t) \in L^{2}(G)$ such that $\left(1-\Delta_{x}\right)^{-1}$ $\partial_{x}^{\nu} \partial_{t}^{j} u(x, t) \in L^{2}(G)$ for any $\nu$ and $j(|\nu|+2 j \leqq 2)$, where we define $\left(1-\Delta_{x}\right)^{-1} v(x, t)$ by $\overline{\mathscr{F}}_{\xi}\left[\left(1+|\xi|^{2}\right)^{-1} \hat{v}(\xi, t)\right]$ and we take the derivatives $\partial_{x}^{\nu} \partial_{t}^{j} u(x, t)$ in the sense of distribution in $G$. $\mathscr{E}_{[-2]}^{2,1}(G)$ provided with the scalar product

$$
(u, v)_{\mathscr{C}_{\left[-2, \frac{1}{2}\right]^{\prime}(G)}}=(u, v)_{L^{2}(G)}+\left(\left(1-\Delta_{x}\right)^{-1} \partial_{t} u,\left(1-\Delta_{x}\right)^{-1} \partial_{t} v\right)_{L^{2}(G)}
$$

is a Hilbert space. Hence $\left\{u^{(k)}(t)\right\}$ has a weak limit, say $u(x, t) . u(x, t)$ satisfies the equation

$$
\partial_{t} u-A u=f \quad \text { in the sense of distribution in } G \text {. }
$$

Now we show that $u(x, t)$ is the solution in the sense of Theorem 1 . 
At first we show that $t \rightarrow u(x, t) \in \mathscr{D}_{L^{2}}^{\prime 2}$ is continuous. Because $\partial_{t}\left(1-\Delta_{x}\right)^{-1} u$ $\in L^{2}(G)$, by Nikodym's theorem, for almost every $x,\left(1-\Delta_{x}\right)^{-1} u$ is an absolutely continuous function of $t$, where if necessary we modify $u(x, t)$ on a subset $(\subset G)$ of measure 0 . Therefore

$$
\left(1-\Delta_{x}\right)^{-1} u\left(x, t_{2}\right)-\left(1-\Delta_{x}\right)^{-1} u\left(x, t_{1}\right)=\int_{t_{1}}^{t_{2}} \partial_{t}\left(1-\Delta_{x}\right)^{-1} u(x, t) d t
$$

for a.e. $x$. By Schwarz' inequality

$$
\begin{aligned}
& \left|\left(1-\Delta_{x}\right)^{-1} u\left(x, t_{2}\right)-\left(1-\Delta_{x}\right)^{-1} u\left(x, t_{1}\right)\right|^{2} \\
& \quad \leqq\left|t_{2}-t_{1}\right| \int_{t_{1}}^{t_{2}}\left|\partial_{t}\left(1-\Delta_{x}\right)^{-1} u(x, t)\right|^{2} d t
\end{aligned}
$$

for a.e. $x$. Integrating for $x$ over $\mathbb{R}^{n}$, we have

$$
\begin{aligned}
& \left\|\left(1-\Delta_{x}\right)^{-1} u\left(x, t_{2}\right)-\left(1-\Delta_{x}\right)^{-1} u\left(x, t_{1}\right)\right\| \\
& \quad \leqq\left|t_{2}-t_{1}\right|^{1 / 2}\left\|\partial_{t}\left(1-\Delta_{x}\right)^{-1} u(x, t)\right\|_{L^{2}(G)}
\end{aligned}
$$

We can also show that $\lim _{\varepsilon \rightarrow 0} u(x, \varepsilon)=u_{0}(x)$ in $L^{2}\left(\mathbb{R}^{n}\right)$. But we don't give the proof. The reader will easily verify by consulting the reasoning in $[3]$ ( $\$ 6$ in Chapter 6).

At last we show $u(x, t) \in \mathscr{E}_{t}^{0}\left(L^{2}\right) \cap \mathscr{E}_{t}^{1}\left(\mathscr{D}_{L^{2}}^{\prime 2}\right)$. Apply Friedrichs' mollifier to (3.9). Then we have

$$
\partial_{t} u_{\varepsilon}-A u_{\varepsilon}=f_{\varepsilon}+\left[\rho_{\varepsilon} *, A\right] u \text {. }
$$

We know that $u_{\varepsilon}(x, t) \in \mathscr{E}_{t}^{0}\left(\mathscr{D}_{L^{2}}^{2}\right)$, because $u(x, t) \in \mathscr{E}_{t}^{0}\left(\mathscr{D}_{L^{2}}^{2}\right)$. Besides

$$
\begin{aligned}
& <u_{\varepsilon}(t)-u_{0}^{\varepsilon}, \varphi>=\int_{\mathbb{R}^{n}} \int_{0}^{t}\left(\partial_{t} u_{\varepsilon}(s)\right) \varphi d s d x \\
& =\int_{\mathbb{R}^{n}} \int_{0}^{t}\left\{A u_{\varepsilon}(s)+f_{\varepsilon}(s)+\left[\rho_{\varepsilon} *, A\right] u(s)\right\} \varphi d t d x, \quad \text { for any } \varphi(x) \in \mathscr{D},
\end{aligned}
$$

where we used the fact that the equation (3.10) is satisfied in the $L^{2}(G)$ sense. Hence

$$
u_{\varepsilon}(t)-u_{0}^{\varepsilon}=\int_{0}^{t}\left\{A u_{\varepsilon}(s)+f_{\varepsilon}(s)+\left[\rho_{\varepsilon} *, A\right] u(s)\right\} d s
$$


as a $L^{2}$-valued function of $\iota$. Thus we see that $u_{\varepsilon}(t) \in \mathscr{E}_{t}^{1}\left(L^{2}\right)$. Therefore we can use the same argument as the one in Proposition 1 and have

$$
\begin{aligned}
\frac{d}{d t}\left\|u_{\varepsilon}(t)-u_{\varepsilon^{\prime}}(t)\right\|^{2} \leqq C\left\|u_{\varepsilon}(t)-u_{\varepsilon^{\prime}}(t)\right\|^{2}+\left\|f_{\varepsilon}(t)-f_{\varepsilon^{\prime}}(t)\right\|^{2} \\
\left.+2 \mid \operatorname{Re}\left(u_{\varepsilon}(t)-u_{\varepsilon^{\prime}}(t),\left[\rho_{\varepsilon}-\rho_{\varepsilon^{\prime}}\right) *, A\right] u(t)\right) \mid .
\end{aligned}
$$

Hence

$$
\begin{aligned}
\max _{0 \leqq t \leqq T} \| u_{\varepsilon}(t)- & u_{\varepsilon^{\prime}}(t) \|^{2} \leqq C^{\prime}\left\{\left\|u_{0}^{\varepsilon}-u_{0}^{\varepsilon^{\prime}}\right\|^{2}+\max _{0 \leqq t \leqq T}\left\|f_{\varepsilon}(t)-f_{\varepsilon^{\prime}}(t)\right\|^{2}\right. \\
& \left.+\int_{0}^{t}\left|\operatorname{Re}\left(u_{\varepsilon}(s)-u_{\varepsilon^{\prime}}(s),\left[\left(\rho_{\varepsilon}-\rho_{\varepsilon^{\prime}}\right) *, A\right] u(s)\right)\right| d s\right\}
\end{aligned}
$$

On the other hand in the same way as Lemma 1 we can show for almost every $s(0 \leqq s \leqq T)$

$$
\begin{aligned}
& \left|\operatorname{Re}\left(u_{\varepsilon}(s)-u_{\varepsilon^{\prime}}(s),\left[\left(\rho_{\varepsilon}-\rho_{\varepsilon^{\prime}}\right) *, A\right] u(s)\right)\right| \leqq \text { const. }\|u(s)\|^{2} \\
& \left|\operatorname{Re}\left(u_{\varepsilon}(s)-u_{\varepsilon^{\prime}}(s),\left[\left(\rho_{\varepsilon}-\rho_{\varepsilon^{\prime}}\right) *, A\right] u(s)\right)\right| \rightarrow 0 \quad \text { as } \varepsilon \rightarrow 0 .
\end{aligned}
$$

Hence, noting that $\int_{0}^{T}\|u(s)\|^{2} d s<+\infty$, by Lebesgue's theorem we see that the right-hand terms of (3.12) tend to 0 as $\varepsilon \rightarrow 0$. Thus $\left\{u_{\varepsilon}(t)\right\}$ is a Cauchy sequence in $\mathscr{E}_{t}^{0}\left(L^{2}\right)(0 \leqq t \leqq T)$, as $\varepsilon \rightarrow 0$. Therefore it follows that $t \rightarrow u(x, t) \in L^{2}$ is continuous. By the same reasoning as (3.11) we know that $t \rightarrow u(x, t) \in \mathscr{D}_{L^{2}}^{\prime 2}$ is continuously differentiable. $\quad$ Q.E.D.

PRemank For simplicity we have assumed that all the coefficients are real-valued functions. Here we consider the case where $b_{j}(x, t)$ and $c(x, t)$ are complex-valued functions. Put $b_{j}=b_{j}^{(1)}+i b_{j}^{(2)}$, where $b_{j}^{(1)}$ and $b_{j}^{(2)}$ are real-valued. We assume the following condition: for any $(x, t) \in \mathbb{R}^{n} \times[0, \infty)$

$$
\left(\sum_{j=1}^{n} b_{j}^{(2)}(x, t) \xi_{j}\right)^{2} \leqq C\left(\sum_{j, k=1}^{n} a_{j k}(x, t) \xi_{j} \xi_{k}\right) \quad \text { for all } \xi \in \mathbb{R}^{n}
$$

where $C$ is a universal constant.

Then Proposition 1, 2, Theorem 1 and Theorem 2 (in the following section) are all valid. To see these we have only to note that

$$
\left|\left(u, \sum_{j=1}^{n} b_{j}^{(2)} \partial_{x_{j}} u\right)\right| \leqq \varepsilon \sum_{j, k=1}^{n}\left(a_{j k} \partial_{x_{k}} u, \partial_{x_{j}} u\right)+C_{\varepsilon}\|u\|^{2},
$$


where $\varepsilon$ is an arbitrary small positive constant and $C_{\varepsilon}$ is a constant dependent on $\varepsilon$.

The above condition (3.13) is, in some sense, close to being necessary. In another paper, the author will publish some results related with the necessary condition.

\section{$\S 4$. Smoothness of the Solution}

In this section we study the smoothness of the solution, assuming the smoothness of $u_{0}(x)$ and $f(x, t)$. Let $a_{j k}(x, t) \in \mathscr{E}_{t}^{0}\left(\mathscr{B}^{m+2}\right) ; b_{j}(x, t) \in$ $\mathscr{E}_{t}^{0}\left(\mathscr{B}^{m+1}\right) ; c(x, t) \in \mathscr{E}_{t}^{0}\left(\mathscr{B}^{m}\right)$, where $m=0,1, \ldots$ We assume the condition (1.3). Then we have

Theorem 2. For any initial value $u_{0}(x) \in \mathscr{D}_{L^{2}}^{m}$ and any $f(x, t) \in$ $\mathscr{E}_{t}^{0}\left(\mathscr{D}_{L^{2}}^{m}\right)$, there exists a unique solution $u(x, t) \in \mathscr{E}_{t}^{0}\left(\mathscr{D}_{L^{2}}^{m}\right) \cap \mathscr{E}_{t}^{1}\left(\mathscr{D}_{L^{2}}^{m-2}\right)$ of the Cauchy problem (1.1)-(1.2).

If we use the following propositions, we can prove the above theorem in the same way as Theorem 1 .

Proposition 3. Let $f(t) \in \mathscr{E}_{t}^{0}\left(\mathscr{D}_{L^{2}}^{m}\right)$ and $u(t) \in \mathscr{E}_{t}^{0}\left(\mathscr{D}_{L^{2}}^{m}\right) \cap \mathscr{E}_{t}^{1}\left(\mathscr{D}_{L^{2}}^{m-2}\right)$ be $a$ solution of (1.1). Then it holds for any $t(0 \leqq t \leqq T)$ that

$$
\|u(t)\|_{m} \leqq e^{\gamma^{\prime} t}\|u(0)\|_{m}+\int_{0}^{t} e^{\gamma^{\prime}(t-s)}\|f(s)\|_{m} d s
$$

where $\gamma^{\prime}$ is a constant which may depend on $T$ but does not depend on $u, f$.

Proposition 4. Assume all the coefficients be functions of only $x$. Take the domain of definition $\mathscr{D}_{m}(A)$ of $A$ as follows:

$$
\mathscr{D}_{m}(A)=\left\{u(x) ; u(x) \in \mathscr{D}_{L^{2}}^{m}, A u(x) \in \mathscr{D}_{L^{2}}^{m}\right\}
$$

Then, for large $\lambda,(\lambda-A)$ defines a one-to-one surjective mapping of $\mathscr{D}_{m}(A)$ onto $\mathscr{D}_{L^{2}}^{m}$. Moreover there exists a constant $\beta$ such that

$$
\left\|(\lambda-A)^{-1}\right\|_{\mathscr{L}\left(\mathscr{D}_{L^{2}}^{m}, \mathscr{D}_{L^{2}}^{m}\right.} \leqq \frac{1}{\lambda-\beta} \quad \text { for any } \lambda>\beta
$$


To see these, the following lemma is essential. It is the improved one of Lemma 1. We denote the dual space of $\mathscr{D}_{L^{2}}^{m}$ by $\mathscr{D}_{L^{2}}^{-m}$. Let $\alpha_{m}(\xi)$ $=1+\sum_{1 \leqq|\nu| \leqq m}(2 \pi \xi)^{2 \nu} . \quad$ We define $\alpha_{m}^{-1} u, \quad \alpha_{m}^{-\frac{1}{2} u}$ by $\overline{\mathscr{F}}\left[\alpha_{m}(\xi)^{-1} \hat{u}(\xi)\right]$, $\overline{\mathscr{F}}\left[\alpha_{m}(\xi)^{-\frac{1}{2}} \hat{u}(\xi)\right]$ respectively. $\mathscr{D}_{L^{2}}^{m}$ provided with the scalar product $(u, v)_{-m}=\left(\alpha_{m}^{-\frac{1}{2}} u, \alpha_{m}^{-\frac{1}{2}} v\right)$ is a Hilbert space.

Lemma 2. Let $\rho_{\varepsilon} *$ be the same one as in Lemma 1. Let $a(x) \in \mathscr{B}^{m+2}$ be a real-valued function, and let $u(x) \in \mathscr{D}_{L^{2}}^{m}$. Then it holds for any $\nu(|\nu| \leqq 2)$ that

1) $\left|\operatorname{Re}\left(u_{\varepsilon},\left[\rho_{\varepsilon} *, a(x)\right] \partial_{x}^{\nu} u\right)_{m}\right| \leqq C\|u\|_{m}^{2}$

2) $\operatorname{Re}\left(u_{\varepsilon},\left[\rho_{\varepsilon}^{*}, a(x)\right] \partial_{x}^{\nu} u\right)_{m} \rightarrow 0 \quad$ as $\varepsilon \rightarrow 0$.

Moreover, if $u \in \mathscr{D}_{L^{2}}^{m}$, it holds for any $\nu(|\nu| \leqq 2)$ that

3) $\left|\operatorname{Re}\left(u_{\varepsilon},\left[\rho_{\varepsilon} *, a(x)\right] \partial_{x}^{\nu} u\right)_{-m}\right| \leqq C^{\prime}\|u\|_{-m}$

4) $\operatorname{Re}\left(u_{\varepsilon},\left[\rho_{\varepsilon} *, a(x)\right] \partial_{x}^{\nu} u\right)_{-m} \rightarrow 0 \quad$ as $\varepsilon \rightarrow 0$,

where $C, C^{\prime}$ are constants independent of $u$ and $\varepsilon$.

Only to the latter half, we give a rough sketch of the proof.

$$
\begin{aligned}
\operatorname{Re}\left(u_{\varepsilon},\left[\rho_{\varepsilon} *, a(x)\right] \partial_{x}^{\nu} u\right)_{-m} & =\operatorname{Re}\left(\alpha_{m}^{-1} u_{\varepsilon},\left[\rho_{\varepsilon} *, a(x)\right] \partial_{x}^{\nu} \alpha_{m} \alpha_{m}^{-1} u\right) \\
& =\operatorname{Re}\left(\alpha_{m}^{-1} u_{\varepsilon},\left[\rho_{\varepsilon} *, a(x)\right] \partial_{x}^{\nu} \alpha_{m}^{-1} u\right)_{m} \\
& +\operatorname{Re}\left(\alpha_{m}^{-1} u_{\varepsilon},\left[\rho_{\varepsilon} *,\left[a(x), \alpha_{m}\right]\right] \partial_{x}^{\nu} \alpha_{m}^{-1} u\right) .
\end{aligned}
$$

Because $\alpha_{m}^{-1} u \in \mathscr{D}_{L^{2}}^{m}$, we see that 3 ) and 4) hold for the 1 -st term by the first half of this lemma. Represent $\left[a(x), \alpha_{m}\right] \partial_{x}^{\nu}$ in the following form:

$$
\left[a(x), \alpha_{m}\right] \partial_{x}^{\nu}=\sum_{\substack{\left|\mu_{1}\right| \leq m \\\left|\mu_{2}\right| \leq m+1}} \partial_{x}^{\mu_{1}} a_{\mu_{1} \mu_{2}}(x) \partial_{x}^{\mu_{2}}, \quad a_{\mu_{1} \mu_{2}}(x) \in \mathscr{B}^{2} .
$$

This representation is not unique, of course. Then

$$
\text { 2-nd term }=\sum_{\left|\mu_{1}\right| \leqq m,\left|\mu_{2}\right| \leqq m+1} \operatorname{Re}\left((-1)^{\left|\mu_{1}\right|} \partial_{x}^{\mu_{1}} \alpha_{m}^{-1} u_{\varepsilon},\left[\rho_{\varepsilon} *, a_{\mu_{1} \mu_{2}}(x)\right] \partial_{x}^{\mu_{2}} \alpha_{m}^{-1} u\right) .
$$

Therefore, by Friedrichs' lemma, we see that 3) and 4) are also true for the 2-nd term. 
Ackmowledgememt. The author wishes to express his sincere thanks to Professor S. Mizohata for valuable suggestions and for the constant encouragement.

\section{References}

[1] Friedrichs, K. O., Symmetric hyperbolic system of linear differential equations, Comm. P. A.M., 7 (1954), 345-392.

[2] Igari, K., Well-posedness of the Cauchy problem for some evolution equations, to appear.

[3] Mizohata, S., Theory of partial differential equations, Iwanami, Tokyo (1965) (in Japanese), to appear in English from Camb. Univ. Press.

[4] Oleynik, O.A., On the smoothness of the solutions of degenerate elliptic and parabolic equations, Sov. M. Dokl., 6 (1965), 972-976.

[5] Oleinnik, O.A., Linear equations of second order with non-negative form, Math. Sbornik, 69 (1966) 111-140, (in Russian). Amer. M.S. Translation, Ser. 2, 65, 167-199. 